Voix et Images

voixetimages

\title{
La guerre dans la littérature québécoise
}

\section{Michel Biron et Olivier Parenteau}

Volume 37, numéro 2 (110), hiver 2012

La guerre dans la littérature québécoise

URI : https://id.erudit.org/iderudit/1008572ar

DOI : https://doi.org/10.7202/1008572ar

Aller au sommaire du numéro

\section{Éditeur(s)}

Université du Québec à Montréal

ISSN

0318-9201 (imprimé)

1705-933X (numérique)

Découvrir la revue

Citer ce document

Biron, M. \& Parenteau, O. (2012). La guerre dans la littérature québécoise. Voix et Images, 37(2), 9-14. https://doi.org/10.7202/1008572ar d'utilisation que vous pouvez consulter en ligne.

https://apropos.erudit.org/fr/usagers/politique-dutilisation/ 


\title{
LA G UERRE DANS LA LITTÉRATURE QUÉBÉCOISE
}

MICHEL BIRON

Université McGill

OLIVIER PARENTEAU

Cégep de Saint-Laurent

\author{
En ces régions d'ancienne seigneurie \\ de vergers séculaires et d'arpents lumineux, \\ le siècle s'écoula sans heurts, \\ on ne vit aucun tank \\ au trécarré des terres arables, \\ ni d'enfant estropié par une mine \\ enfouie dans les labours ${ }^{1}$
}

Les rapports entre la guerre et la littérature québécoise n'ont été traités jusqu'ici par la critique que de façon indirecte. On s'est beaucoup intéressé, par exemple, au boom éditorial consécutif à la capitulation de la France en mai 1940 et aux deux crises de la conscription (1917 et 1942), qui sont des dates clés de l'histoire littéraire du Québec. Mais qu'en est-il des textes eux-mêmes ? Comment la guerre est-elle représentée dans les romans, les poèmes, les pièces de théâtre ou les essais? La question peut sembler piégée dans la mesure où les différentes manières de raconter, de penser ou d'imaginer la guerre ne sont nullement spécifiques au corpus québécois et, par là, ouvrent le grand récit national à un contexte transnational. Or c'est là justement leur intérêt, nous semble-t-il : l'imaginaire de la guerre tend à déterritorialiser la littérature québécoise. Mis à part les rébellions de 1837-1838 qui deviennent le symbole de ce qu'Hubert Aquin appellera "l'art de la défaite ${ }^{2}$ ", mis à part aussi quelques vieux poèmes patriotiques comme Le drapeau de Carillon ${ }^{3} \mathrm{~d}^{\prime}$ Octave Crémazie ou des textes plus modernes liés à la conscription, la guerre a lieu ailleurs, sur un autre continent, et déplace le grand texte national. C'est la guerre des autres, parfois même la guerre de l'autre, de l'adversaire par excellence de la nation, c'est-à-dire des Anglais. Dans tous les cas, les guerres du XXe siècle ne cadrent pas avec le discours de l'enracinement auquel on associe l'essor de la littérature québécoise.

1 Pierre Nepveu, Lignes aériennes, Montréal, Éditions du Noroît, 2002, p. 37. 2 Hubert Aquin, «L'art de la défaite. Considérations stylistiques", Liberté, vol. VII, $n^{\text {os }} 1-2$, janvier-avril 1965, p. 33-41. 3 Octave Crémazie, Le drapeau de Carillon. Hommage à MM. les abonnés du Journal de Québec, Québec, [s.n.], 1858, 8 p. 
Dans son poème «Le jeu », Saint-Denys Garneau résume bien le sentiment d'extériorité que suscitent les guerres du monde:

Et pourtant dans son œil gauche quand le droit rit

Une gravité de l'autre monde s'attache à la feuille d'un arbre

Comme si cela pouvait avoir une grande importance

Avait autant de poids dans sa balance

Que la guerre d'Éthiopie

Dans celle de l'Angleterre ${ }^{4}$.

D'octobre 1935 à mai 1936, l'Italie de Mussolini mène une offensive pour s'emparer de l'Éthiopie pendant que l'Angleterre, comme les autres pays de la Société des Nations, assiste, impuissante, à ce qui marque l'engagement de l'Italie fasciste envers l'Allemagne nazie. Le poète ne sait pas que cette guerre deviendra mondiale deux ans plus tard et, de toute façon, il ne se réfère pas à la guerre comme on le fait dans les journaux et à la radio de l'époque. La gravité qui se voit dans son œil gauche tandis que le droit s'amuse transcende le contexte historique: c'est une gravité «de l'autre monde», c'est-à-dire du monde de l'au-delà, mais elle trouve une image forte dans une actualité qui envahit les consciences et qui projette le ludisme de l'enfant dans l'absurdité des drames universels.

Ces guerres ont beau avoir lieu sur d'autres continents, elles fascinent l'écrivain canadien-français malgré la distance ou, plus exactement, elles le fascinent par la distance même qui le sépare de l'événement. Celle-ci a longtemps été compensée par un patriotisme romantique dont on trouve encore des traces au moment de la Première Guerre mondiale. L'exemple le plus connu est sans doute le recueil Lauriers et feuilles d'érable (1916) d'Albert Lozeau, qui en montre des signes dès le poème liminaire, "Le drapeau», où le soldat canadien-français se bat au nom du drapeau français, celui de sa mère patrie:

\footnotetext{
Qu'importe son tissu vieux ou neuf, soie ou toile,

C'est le suprême signe et l'emblème sacré !

Le soldat dit: «Pour lui, noblement, je mourrai,

Car il est mon chemin, mon guide et mon étoile ${ }^{5}$ !»
}

On n'écrira plus guère de cette façon à partir du moment où la poésie, comme l'ensemble de la littérature canadienne-française, refusera ce romantisme qui rapproche le poème d'un discours de propagande. L'écrivain parlera depuis sa propre expérience du monde plutôt qu'au nom de valeurs abstraites, de lieux communs et de mots empruntés.

Même si les grands conflits guerriers du Xx siècle se déroulent loin du territoire québécois, ils n'en jouent pas moins un rôle majeur dans l'avènement de la modernité culturelle. C'est le cas tout particulièrement de la Seconde Guerre mondiale, qui

$$
+++
$$

4 Saint-Denys Garneau, «Le jeu », E Euvres, texte établi, annoté et présenté par Jacques Brault et Benoît Lacroix, Montréal, Presses de l’Université de Montréal, coll. «Bibliothèque des lettres québécoises », 1971, p. 11. 5 Albert Lozeau, Lauriers et feuilles d'érable, Montréal, Édition du Devoir, 1916, p. 7. 
marque un tournant important dans l'histoire littéraire du Québec. En 1945, Alain Grandbois choisit par exemple de donner à un recueil de nouvelles écrites avant la guerre le titre Avant le chaos, qui en remotive le sens a posteriori. Il s'en explique dans son avant-propos:

Les temps pleins d'angoisse que nous traversons nous défendent d'ajouter aux profondeurs du drame la note sacrilège des jeux de l'insouciance et de la désinvolture. Aussi dois-je sauter par-dessus cinq ans de guerre pour rejoindre une époque singulièrement révolue. Les hommes de ma génération ont vécu des jours que leurs cadets ne connaîtront jamais ${ }^{6}$.

Grandbois est rentré au pays à cause de la guerre, et ses textes ne parleront donc pas de la guerre vécue sur le front. Mais les nouvelles qu'il a écrites avant 1939 trouvent une résonance nouvelle après la guerre, tout comme l'ensemble des textes littéraires parus au Québec au milieu du siècle. La plupart mettent en scène d'effrayants drames intérieurs, comme si l'individu était marqué par une crise des valeurs et par un pessimisme généralisé à l'égard du monde.

Dans les rares récits faisant directement référence à la Seconde Guerre mondiale, comme Neuf jours de haine de Jean-Jules Richard, ce pessimisme passe par le témoignage cru du journal de bord: «Il pleut. La cinquième dimension suinte son oxygène. Son oxygène est humide. On s'est aligné sur le bord d'une rivière. Des avions ennemis viennent. On est crispé. Pas de terriers. C'est terrible. Où s'abriter? Nulle part. On peut seulement s'abriter sur la terre. C'est terrible ${ }^{7}$.» De même pour Les Canadiens errants ${ }^{8}$, roman-témoignage de Jean Vaillancourt, qui n'écrira aucun autre livre. La guerre y est décrite au quotidien, mais aussi le retour au pays. Lorsque le héros des Canadiens errants, un mercenaire qui n'a jamais rien fait d'autre que la guerre dans sa vie, revient à Montréal après avoir été blessé à la jambe, le monde civil lui est devenu totalement étranger. Ici, le thème de l'aliénation, si central dans le roman canadien-français du milieu du siècle, croise un des leitmotive du roman de guerre, de Barbusse à Aragon, celui de l'impossible adaptation à la vie civile après l'expérience du front:

\footnotetext{
Son véritable et unique mal était sa solitude. On n'avait pas idée de sortir de l'Armée quand on avait un passé comme le sien. D'où venait-il? Il se sentait comme un de ces étrangers météorites, détachés un jour de leur planète, égarés dans les ténèbres d'un espace incommensurable et condamnés à errer dans ce néant ${ }^{9}$.
}

Pour saisir de quelle façon la guerre entre dans la conscience des individus qui vivent loin du front, à un océan de distance, il suffit de relire la magnifique scène de Bonheur d'occasion où Gabrielle Roy décrit la réalité de la guerre vue d'ici. L'événement prend

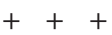

6 Alain Grandbois, Avant le chaos et autres nouvelles, Montréal, Presses de l'Université de Montréal, coll. «Bibliothèque du Nouveau Monde», 1991 [1945], p. 45.7 Jean-Jules Richard, Neuf jours de haine, Montréal, Éditions de l'Arbre, 1948, p. 85. 8 Jean Vaillancourt, Les Canadiens errants, Montréal, Le Cercle du livre de France, 1954, 250 p. 9 Ibid., p. 262. 
forme à travers les yeux incrédules de Rose-Anna, qui songe tout à coup à son fils enrôlé lorsqu'elle apprend, par les journaux, que l'Allemagne vient d'envahir la Norvège :

Elle resta hébétée un moment, l'œil dans le vide, et tirant sur la courroie de son sac. Elle ne sut pas d'abord d'où lui venait le coup qui la paralysait. Puis, dressée au malheur, sa pensée vola vers Eugène. De quelque façon inexplicable et dure, elle crut sur l'instant que le sort de son fils dépendait de cette nouvelle. Elle relut les gros caractères, syllabe par syllabe, formant à demi les mots du bout de ses lèvres. Sur le mot «Norvège», elle s'arrêta pour réfléchir. Et ce pays lointain, qu'elle ne savait situer que vaguement, lui parut lié à leur vie d'une manière définitive et incompréhensible. Elle n'examina, ne calcula, ne pesa rien, elle oublia qu'Eugène l'assurait, dans sa dernière lettre, qu'il resterait au moins six mois dans le camp d'entraînement. Elle voyait des mots qui s'allongeaient devant elle lourds de danger immédiat. Et cette femme qui ne lisait que son livre d'heures fit une chose extraordinaire. Elle traversa rapidement la chaussée en fouillant dans son sac à main; et à peine arrivée sur le trottoir d'en face, elle tendit trois sous au vendeur de journaux et déplia aussitôt la gazette humide qu'il lui avait remise. S'appuyant au mur d'un magasin, elle lut quelques lignes, poussée, entraînée par des ménagères qui sortaient de la fruiterie, et retenant son sac comme elle le pouvait sous son bras serré contre elle. Au bout d'un moment, elle plia le journal d'un geste absent, et leva devant elle des yeux lourds de colère [...].

D'un pas d'automate, elle prit le chemin de la rue Beaudoin. Elle les connaissait bien, soudain, toutes ces femmes des pays lointains, qu'elles fussent polonaises, norvégiennes ou tchèques ou slovaques. C'étaient des femmes comme elle. Des femmes du peuple. Des besogneuses [...]. Elle était de celles qui n'ont rien d'autre à défendre que leurs hommes et leurs fils. De celles qui n'ont jamais chanté aux départs. De celles qui ont regardé les défilés avec des yeux secs et, dans leur cœur, ont maudit la guerre. [...] Elle voulut se reprendre, se défendre de la haine comme de la pitié. «On est au Canada, se disait-elle en brusquant le pas; c'est bien de valeur ce qui se passe làbas, mais c'est pas de notre faute.» Elle reniait farouchement ce cortège triste qui l'accompagnait au retour. Mais elle ne pouvait aller assez vite pour s'en dégager. Une foule innombrable l'avait rejointe, venant mystérieusement du passé, de tous les côtés, de très loin et aussi de très près, semblait-il, car des visages nouveaux surgissaient à chaque pas, et ils lui ressemblaient ${ }^{10}$.

Tel est le grand choc de la guerre : le monde extérieur le plus éloigné (cette Norvège que Rose-Anna ne sait même pas où situer au juste sur une carte) fait brusquement irruption dans les mentalités des gens les moins voyageurs, les moins intellectuels, les moins préparés à affronter une telle rencontre. Les journaux et la radio abolissent les distances de sorte que la Canadienne française Rose-Anna devient solidaire des femmes polonaises, norvégiennes, tchèques ou slovaques. La guerre élargit la conscience et force le regard au-delà de la nation, vers l'universel.

$$
++
$$

10 Gabrielle Roy, Bonheur d'occasion, Montréal, Boréal, coll. «Boréal compact», 1993 [1945], p. 239-240. 
Ce regard qui s'ouvre un peu malgré lui à l'international ne peut que percevoir du même coup ce qui sépare la vie et l'histoire canadiennes-françaises des cataclysmes qui s'abattent ailleurs. Chez Hector de Saint-Denys Garneau comme chez Gabrielle Roy, l'actualité de la guerre ressemble à un exercice de géographie et renvoie à des réalités dont on n'avait jamais entendu parler jusque-là. En France, il suffit de dire «Verdun» pour qu'émergent des images, des récits, des souvenirs communs. Au Québec, il n'y a pas de tels «lieux de mémoire» associés à la guerre. Le seul souvenir collectif rattaché aux deux guerres mondiales, on l'a dit, est celui de la conscription, qui trouve immédiatement son sens dans le grand récit national opposant les Canadiens français aux Canadiens anglais.

Le plus souvent, ce n'est pas la guerre elle-même qui est racontée, mais plutôt le retour au pays. Ce retour est parfois douloureux, comme en témoigne la colère de Tit-Coq ${ }^{11}$, conscrit puis trahi par Marie-Ange qui ne l'a pas attendu pour se marier. De la guerre elle-même, on ne saura rien. Y a-t-il seulement eu expérience de combat? Pas dans le cas du Simple soldat ${ }^{12}$ de Marcel Dubé, qui s'était engagé dans l'armée sans jamais avoir la «chance» de se battre, la guerre s'étant terminée avant qu'il ne soit envoyé au front. Pour sauver les apparences, il s'invente quelques histoires de guerre auxquelles cependant personne ne croit. La guerre est pour ce soldat raté une pure fiction, un mensonge de plus. Humilié par sa famille, il s'enfuira finalement en s'engageant dans la guerre de Corée, où il mourra sur le champ de bataille. Une décennie plus tard, dans La guerre, yes sir ! ${ }^{13}$ de Roch Carrier, on assiste au rapatriement du corps d'un soldat canadien-français. Les habitants du village d'où vient le défunt se réunissent pour veiller le corps, dans un rituel carnavalesque, sous le regard scandalisé des Anglais. Dans chacune de ces œuvres, la guerre ressemble à une fiction lointaine que l'écrivain tente d'intégrer à la trame nationale.

L'effet de déterritorialisation est encore plus marqué dans la littérature québécoise contemporaine, à l'ère de la mondialisation. Quelques écrivains d'origine étrangère installés au Québec évoquent dans leurs œuvres la guerre qu'ils ont fuie. C'est le cas du dramaturge d'origine libanaise Wajdi Mouawad, notamment dans sa pièce Incendies ${ }^{14}$. Au même moment, plusieurs écrivains d'origine québécoise font le mouvement inverse, allant vers des régions du monde où sévit la guerre pour interroger les gens sur place et recueillir leurs témoignages. En 2000, Madeleine Gagnon publie des entrevues avec des femmes victimes de la guerre dans Les femmes et la guerre ${ }^{15}$, et le journaliste Gil Courtemanche se sert du roman pour décrire la tragédie du génocide rwandais dans Un dimanche à la piscine à Kigali ${ }^{16}$. Pendant que les médias électroniques transportent les drames de partout au cœur de chaque maison, la distance entre ce qui se passe ailleurs et ici tend à diminuer, et les romans qui font référence aux grands conflits se multiplient, de Pierre. La guerre du printemps $81^{17}$ de Marie-

$$
++
$$

11 Gratien Gélinas, Tit-Coq, Montréal, TYPO, coll. «Théâtre», 2010 [1948], 168 p. 12 Marcel Dubé, Un simple soldat, Montréal, TYPO, coll. «Théâtre», 2011 [1957], 200 p. 13 Roch Carrier, La guerre, yes sir !, Montréal, Éditions du Jour, coll. «Les romanciers du jour», 1968, 124 p. 14 Wajdi Mouawad, Incendies, Montréal/Arles, Leméac/Actes Sud, coll. «Actes Sud-Papiers», 2003, 92 p. 15 Madeleine Gagnon, Les femmes et la guerre, Montréal, VLB éditeur, 2000, 306 p. 16 Gil Courtemanche, Un dimanche à la piscine à Kigali, Montréal, Boréal, 2000, 283 p. 17 MarieClaire Blais, Pierre. La guerre du printemps 81, Montréal, Primeur, coll. «L'échiquier», 1984, 165 p. 
Claire Blais ou Omaha Beach. Un oratorio ${ }^{18}$ de Catherine Mavrikakis jusqu'au tout récent roman pour les jeunes, intitulé Guerres ${ }^{19}$, de Charlotte Gingras.

Il est bien sûr impossible de rendre compte de façon globale d'une telle production, et le dossier qui suit ne vise pas un tel objectif. Il s'agit, plus modestement, d'aborder cette vaste question à partir de six études permettant de voir comment la guerre a marqué de diverses façons l'histoire littéraire du Québec. Dans la première étude, Micheline Cambron développe l'hypothèse selon laquelle les faits liés à la Première Guerre mondiale s'inscrivent dans le discours (littéraire et journalistique) « comme des éléments qui témoigneraient d'une mémoire individuelle privée d'horizon général ${ }^{20}$ ». L'hypothèse vaut autant pour le discours élaboré durant la guerre que pour ses représentations ultérieures dans la littérature québécoise, notamment chez Ringuet et Robert de Roquebrune. À partir de documents inédits, Yvan Lamonde fait (re)découvrir la figure du père Marie-Alain Couturier, défenseur de l'art abstrait, et rappelle ainsi à quel point la période de la Seconde Guerre mondiale coïncide, au Québec, avec une effervescence intellectuelle inédite. L'étude d'Élisabeth NardoutLafarge compare Les Canadiens errants à un étonnant roman contemporain publié par le journaliste Richard Hétu en hommage à Jean Vaillancourt sous le titre Rendez-vous à l'Étoile (2006). Elle montre comment le récit fragmenté de Vaillancourt, typique du récit de guerre selon Jean Kaempfer, est repris, mais aussi ordonné dans un roman qui, en cherchant à expliquer les faits et gestes du personnage principal, est orienté vers une visée pédagogique et éthique. Du côté de la poésie, Olivier Parenteau interroge le corpus des années 1960, plus particulièrement les textes de Paul-Marie Lapointe, de Fernand Ouellette et de Jacques Brault, et met en évidence leur recours à de saisissantes images de la guerre. Ces images renvoient à la question du pays, mais elles la complexifient et la transportent dans un temps préhistorique, celui de l'homme primitif. Les deux dernières études portent sur la période plus contemporaine. Yan Hamel s'intéresse au corpus de la littérature destinée aux jeunes et montre à quel point les représentations de la guerre, très fréquentes dans ce type de textes, sont de puissants révélateurs de ce que nous voulons être et permettent de dégager «l'idée que le Québec contemporain se fait de sa propre identité collective ${ }^{21} »$. Enfin, Lucie Robert étudie la trilogie du dramaturge André Ricard composée de La longue marche dans les Avents (1983), du Tréteau des apatrides (1995) et de Gens sans aveu (2008), où sont évoquées aussi bien la guerre de Sept Ans (1756-1763) et les rébellions des Patriotes en 1837-1838 que la Seconde Guerre mondiale.

On voit bien, par l'ensemble de ce dossier, à quel point la question de la guerre, dans un Québec "si monstrueusement en paix ${ }^{22}$ " selon l'expression polémique de Wajdi Mouawad, ne cesse de hanter les écrivains et de marquer l'imaginaire collectif.

$$
++
$$

18 Catherine Mavrikakis, Omaha Beach. Un oratorio, Montréal, Héliotrope, 2008, 123 p. 19 Charlotte Gingras, Guerres, Montréal, La courte échelle, 2011, 152 p. 20 Voir Micheline Cambron, «Le discours sur la Grande Guerre. Demande d'histoire», dans le présent dossier, p. 15-33. 21 Voir Yan Hamel, «La guerre pour la jeunesse», dans le présent dossier, p. 83-94. 22 Cité dans Luc Boulanger, «Wadji Mouawad : quand les hommes vivront d'amour", Voir, 3 octobre 2001, en ligne : http://voir.ca/scene/2001/10/03/wajdi-mouawad-quand-leshommes-vivront-damour/(page consultée le 5 février 2012). 